\title{
Stress Analysis for Asphalt Concrete Pavement of Curved Box Girder \\ Bridge under Interlaminar Contact Condition
}

\author{
Xuntao Wang ${ }^{1, a}$, Jianhu Feng ${ }^{1, b}$, Hu Wang ${ }^{1, c}$ \\ ${ }^{1}$ School of Science, Chang'an University, Xi'an Shaanxi, 710064, China \\ ãEmail:wxt5288@126.com, bemail: jhfeng@chd.edu.cn, email: wh89@chd.edu.cn
}

\begin{abstract}
Keywords: curved box girder bridges, asphalt concrete pavement, interlaminar contact, stress
Abstract: A curved box girder bridge was simulated by ANSYS software, and the interlaminar contact form of deck pavement was achieved by the contact element and target element. Stresses of the asphalt concrete pavement were calculated and gained when material parameters were changed and interlaminar disengaging area was changed. The results showed that: when the thickness of the upper layer of asphalt concrete increased, the most stresses of the asphalt concrete all decreased, and reasonable thickness of asphalt concrete was gained; when the elastic module of the waterproof increased, the most stresses of the asphalt concrete all decreased, so waterproof of high elastic modulus was suggested to be used in practical engineering; when disengaging area occurred between two adjacent layers and vehicle load located on the layer, the stresses of the asphalt concrete all increased; the size and position of disengaging area had great influence on the stresses of asphalt concrete.
\end{abstract}

\section{Introduction}

The curved bridge is a kind of common bridge, it can easily overcome the constraint of geographical environment and therefore it is widely used in expressway and urban overpass [1]. As vehicle load and rainwater acted repeatedly on deck pavement of curved bridge, various kinds of diseases of asphalt concrete pavement emerged gradually, such as: transverse crack, longitudinal crack [2,3] and disengaging [4] between two adjacent layers. For protecting deck pavement from destruction, ample researches [5,6] about asphalt concrete pavement had been done, however, many studies [7,8] had not taken the weight of concrete bridge and the weight of deck pavement into account, the effect of rebar was also ignored in calculation, and the relation $[9,10]$ of paving layer and its adjacent layer(s) was assumed to be completely continuous, these are not reasonable or actual. Although these previous researches simplified calculation model and gained some useful conclusions, its results produced discrepancy with practical engineering. Meanwhile, little researches results about asphalt concrete pavement of curved box girder bridges had been reported, so it was indispensable to analysis the stress of the asphalt concrete pavement of curved box girder bridges, and gained valuable conclusions for practical engineering. In this paper, those defects mentioned above in other studies would be made up, the contact model between paving layers could be used to improve the calculation results, and making the calculation more effective and more practical.

\section{Material Parameters and Computation Model}

Bituminous mixture, cement concrete and rebar were assumed to be continuous, homogeneous, isotropic and linear elastic materials at normal temperature. The asphalt concrete pavement was 
paved after the curved box girder bridge was built completely. The weight of curved box girder, rebar, leveling course, waterproof layer and asphalt concrete pavement were taken into account in the calculation. The material parameters are showed in table 1.

A curved box girder bridge was simulated by ANSYS software, and the structure size of it was shown in figure 1 and figure 2 . The curved box girder, leveling course, the upper layer of asphalt concrete and the lower layer of asphalt concrete were simulated by the 8 nodes solid element, the waterproof layer was simulated by shell element, and the rebar was simulated by link element. The relation between the roof of the curved box girder and leveling course was assumed to be continuous, so the deformation between the roof of the curved box girder and leveling course was consistent. The relation between leveling course and waterproof layer was presumed to be contact with each other, the relation between the waterproof layer and the lower layer of asphalt concrete was presumed to be contact with each other, the relation between the lower layer of asphalt concrete and the upper layer of asphalt concrete was also presumed to be contact with each other. The contact relation between different layers could be achieved by producing the contact element and the target element on the surface of each layer in ANSYS, meanwhile the contact element and target element would come in pair.

Table 1 Material Parameters

\begin{tabular}{ccccc}
\hline Structural layer & Thickness[m] & $\begin{array}{c}\text { Elastic } \\
\text { modulus[MPa] }\end{array}$ & Poisson's ratio & Density[kg/m3] \\
\hline $\begin{array}{c}\text { the upper layer of } \\
\text { asphalt concrete }\end{array}$ & 0.06 & 1800 & 0.25 & 2370 \\
\hline $\begin{array}{c}\text { the lower layer of } \\
\text { asphalt concrete }\end{array}$ & 0.04 & 1200 & 0.25 & 2350 \\
\hline waterproof layer & 0.002 & 150 & 0.3 & - \\
\hline leveling course & 0.1 & 30000 & 0.15 & 2450 \\
\hline rebar & - & 200000 & 0.3 & 7800 \\
\hline curved box girder & - & 34500 & 0.15 & 2559.1 \\
\hline
\end{tabular}

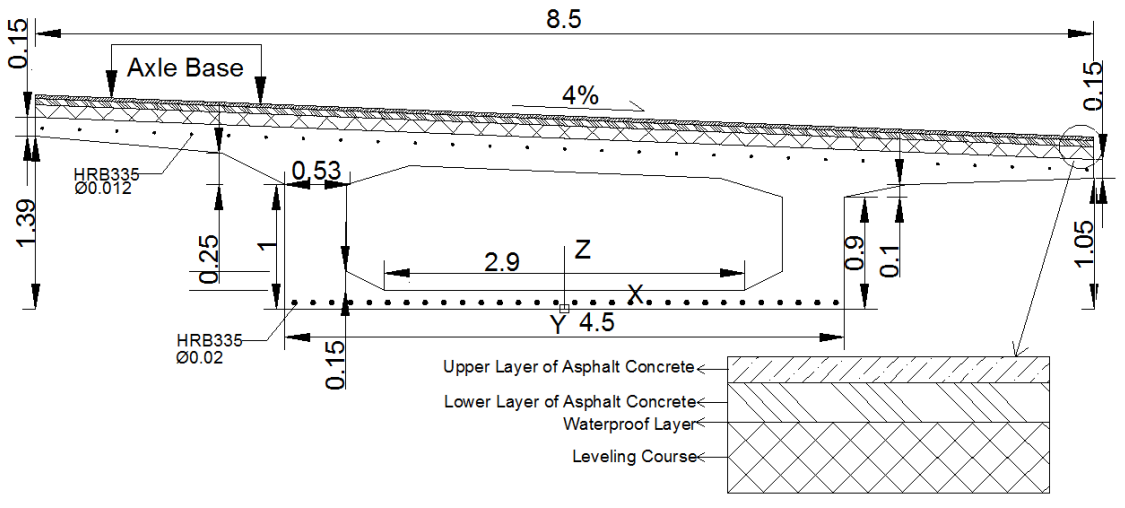

Fig.1 Crossing Section of the Curved Box Girder Bridge (Unit: m) 


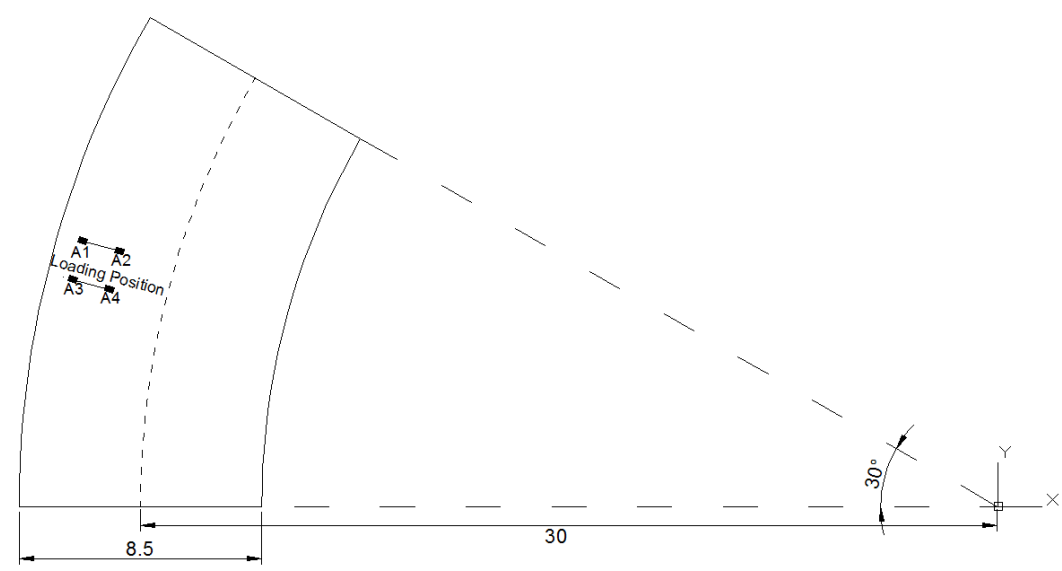

Fig.2 Top View of the Curved Box Girder Bridge (Unit: m)

When a certain layer and its adjacent layer(s) were assumed to be contact with each other, the shear stresses transmission between the adjacent layers follows Coulomb friction model [11]:

$$
\begin{gathered}
\tau_{\lim }=\mu P+b \\
|\tau| \leq \tau_{\lim }
\end{gathered}
$$

Where $\tau_{\lim }$ is the ultimate shear stress; $\mu$ is the sliding friction coefficient, it equals 0.5 in this paper; $P$ is the contact compressive stress in normal direction; $b$ is the cohesion between the adjacent layer; $\tau$ is the equivalent shearing stress. In equation (1), if $\mu$ equals 0 or $P$ equals 0 , the cohesion $b$ still exist; if $b$ equals 0 , two adjacent layers take place cohesive failure. In inequality (2), when $|\tau|$ between two adjacent layers is less than or equal $\tau_{\text {lim }}$, the two layers keep sticking, or the two layers start to slide.

\section{Load Position and Calculated Stresses}

The most unfavorable load position of the curved box girder bridge is showed in the figure 2 . The rear-axle load (double-axle) of the heavy-duty vehicle is $140 \mathrm{KN} \times 2$ [12], and the load region of single-axle load is $0.6 m \times 0.2 m+0.6 m \times 0.2 m$. The area of load position in figure 2 from A1 to A4 all are $0.6 m \times 0.2 m$, and with a tire pressure of $0.7 \mathrm{Mpa}$. When emergency braking occurs, the braking force $F$ acting on the upper layer of asphalt concrete will come into being. In this paper the braking force $F=\lambda G$, where $\lambda$ is the braking coefficient, it equals $0.5 ; G$ is the wheel load. Differing from passing straight bridge, when vehicles pass curved bridge with a constant speed, the stresses of asphalt concrete were also affected by centrifugal force $F_{c}$. The centrifugal force $F_{c}=m v^{2} / r$ in circular motion, where $m$ is object mass; $v$ is object speed, it was presumed to be $10 \mathrm{~m} / \mathrm{s}$ in this paper; $r$ is radius of circle. The stresses $\sigma_{x \max }, \sigma_{y \max }, \sigma_{z \max }, \tau_{x y \max }, \tau_{y z \max }$ and $\tau_{x z \max }$ of asphalt concrete were calculated when material parameters changing and interlaminar disengaging area changing. 


\section{Stresses calculation and stresses analysis for asphalt concrete pavement}

The upper layer of asphalt concrete (or ULAC for short), the lower layer of asphalt concrete (or LLAC for short) and the waterproof layer (or WL for short) are the main part of pavement layer, their thickness and elastic modulus are important material parameters in calculation.

Influence of thickness changing on stresses of asphalt concrete pavement

The stresses value of the ULAC when thickness changing were shown in fig 3. From fig 3A, when the thickness of the ULAC increased from $0.02 \mathrm{~m}$ to $0.08 \mathrm{~m}$, the shear stress $\tau_{y z \max }$ of the ULAC decreased firstly, and then increased, other stresses of the ULAC all decreased with the increasing thickness of the ULAC. From fig 3B, when the thickness of the LLAC increased from $0.03 \mathrm{~m}$ to $0.09 \mathrm{~m}$, the tensile stress $\sigma_{y \max }$ and shear stress $\tau_{x y \max }$ of the ULAC decreased, the tensile stress $\sigma_{z \max }$, shear stress $\tau_{y z \max }$ and $\tau_{x z \max }$ of the ULAC all increased, the tensile stress $\sigma_{x \max }$ of the ULAC decreased firstly, and then increased. When the thickness of the WL increased from $2 \mathrm{~mm}$ to $8 \mathrm{~mm}$, the stresses of the ULAC were almost unchanged.
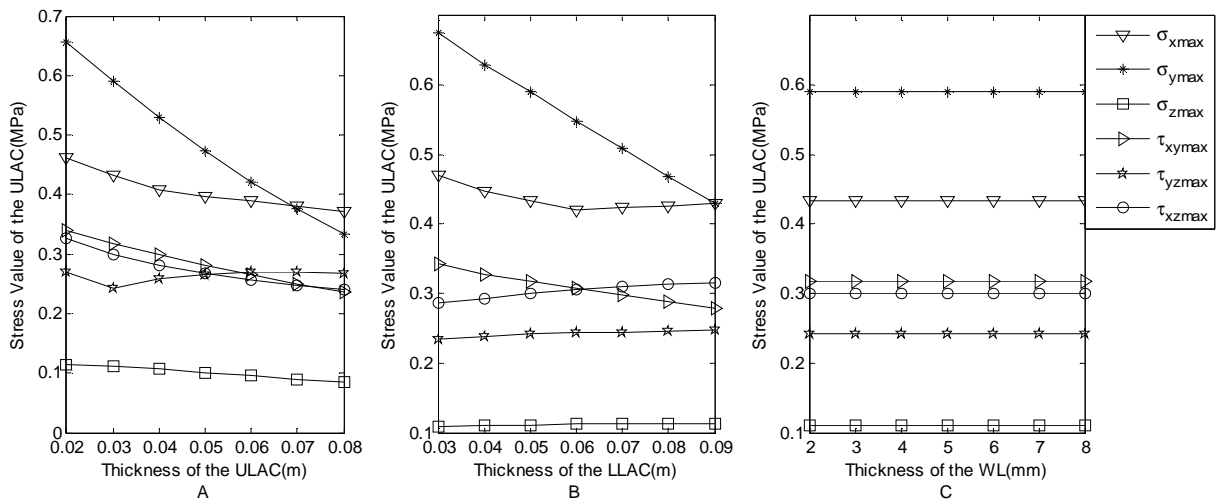

Fig.3 stress value of the ULAC when thickness changing
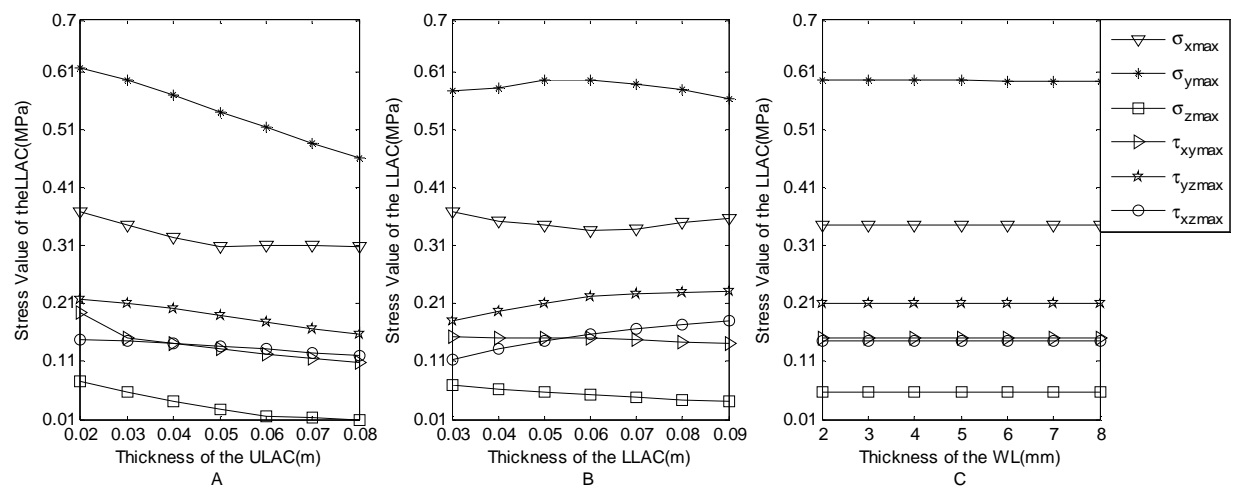

Fig.4 stress value of the LLAC when thickness changing

The stresses value of the LLAC when thickness changing were shown in fig 4. From fig 4A, when the thickness of the ULAC increased from $0.02 \mathrm{~m}$ to $0.08 \mathrm{~m}$, stress of the LLAC all decreased. From fig 4B, when the thickness of the LLAC increased from $0.03 \mathrm{~m}$ to $0.09 \mathrm{~m}$, the tensile stress $\sigma_{x \max }$ of the LLAC decreased firstly, and then increased, the tensile stress $\sigma_{y \max }$ of the LLAC increased firstly, and then decreased, the tensile stress $\sigma_{z \max }$ and shear stress $\tau_{x y \max }$ of the LLAC all 
decreased, the shear stress $\tau_{y z \max }$ and $\tau_{x z \max }$ of the LLAC increased. From fig $4 \mathrm{C}$, when the thickness of the WL increased from $2 \mathrm{~mm}$ to $8 \mathrm{~mm}$, the stresses of the LLAC were almost unchanged.

When the thickness of the ULAC increased, the most stresses of the ULAC and LLAC all decreased; when the thickness of the LLAC increased, the part stresses of ULAC and LLAC increased, the part stresses of them decreased; when the thickness of the WL increased, the stresses of the ULAC and LLAC were almost unchanged.

Influence of elastic modulus changing on stresses of asphalt concrete pavement

The stresses value of the ULAC when elastic modulus changing were shown in fig 5. From fig $5 \mathrm{~A}$, when the elastic modulus of the ULAC increased from $1.1 \mathrm{GPa}$ to $1.7 \mathrm{GPa}$, the tensile stress $\sigma_{x \max }, \sigma_{y \max }$ and shear stress $\tau_{x y \max }$ of the ULAC increased, the tensile stress $\sigma_{z \max }$, shear $\operatorname{stress} \tau_{y z \max }$ and $\tau_{x z \max }$ of the ULAC were almost unchanged. From fig $5 \mathrm{~B}$, when the elastic modulus of the LLAC increased from $0.9 \mathrm{GPa}$ to $1.5 \mathrm{GPa}$, the tensile stress $\sigma_{x \max }, \sigma_{y \max }$ and shear $\operatorname{stress} \tau_{x y \max }$ of the ULAC decreased, the shear stress $\tau_{y z \max }$ and $\tau_{x z \max }$ of the ULAC increased, the tensile stress $\sigma_{z \max }$ of the ULAC were almost unchanged. From fig 5C, when the elastic modulus of the WL increased from $0.15 \mathrm{GPa}$ to $0.45 \mathrm{GPa}$, the tensile stress $\sigma_{x \max }, \sigma_{y \max }$, shear stress $\tau_{x y \max }$ of the ULAC all decreased, the shear stress $\tau_{y z \max }$ of the ULAC increased slightly, the tensile stress $\tau_{x z \max }$ of the ULAC decreased slightly, the shear stress $\sigma_{z \max }$ of the ULAC was almost unchanged.
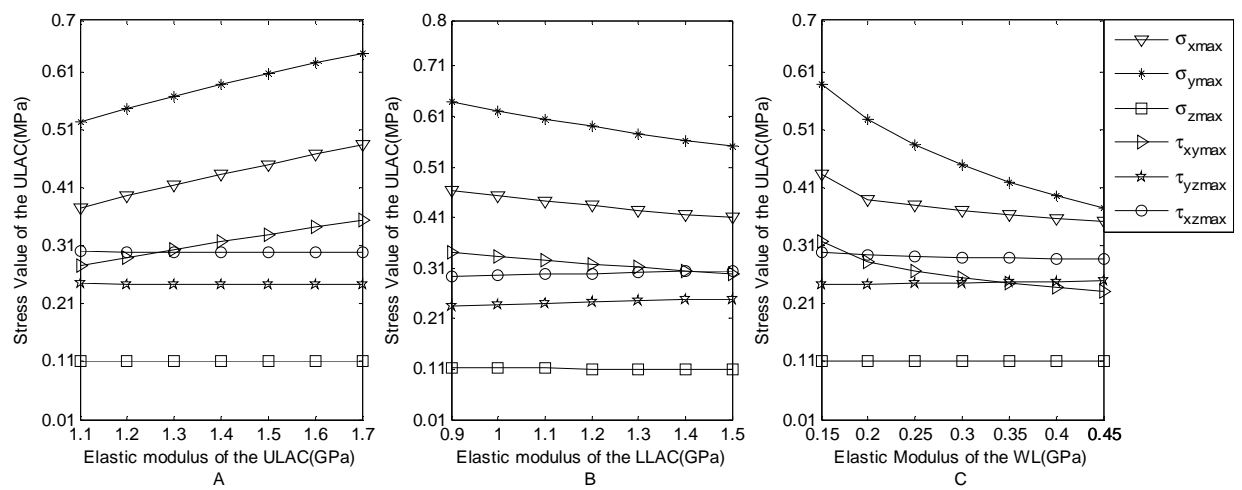

Fig.5 stress value of the ULAC when elastic modulus changing 

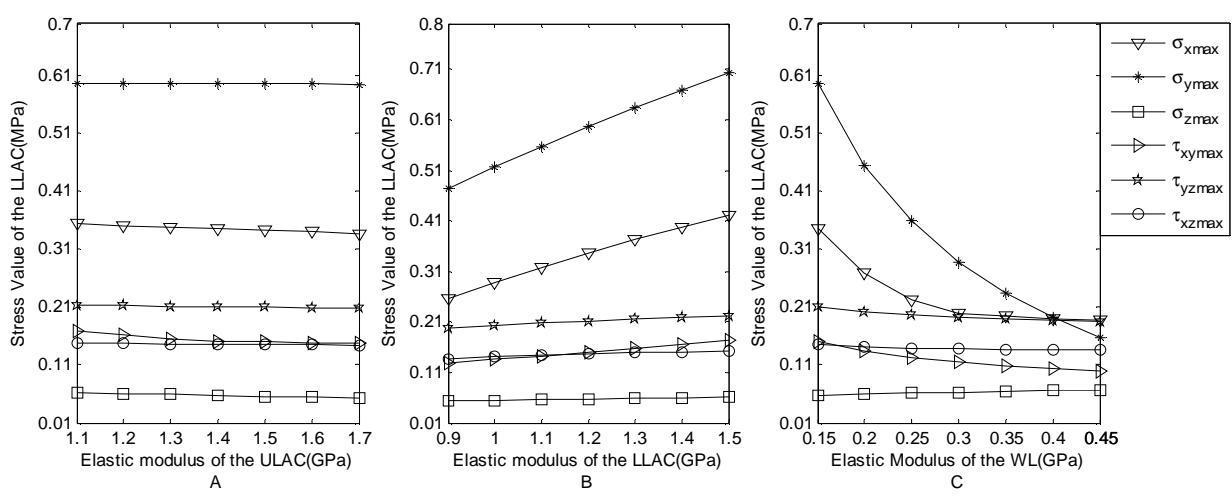

Fig.6 stress value of the LLAC when elastic modulus changing

The stresses value of the LLAC when elastic modulus changing were shown in fig 6. From fig $6 \mathrm{~A}$, when the elastic modulus of the ULAC increased from $1.1 \mathrm{GPa}$ to $1.7 \mathrm{GPa}$, the stresses of the LLAC all decreased. From fig 6B, when the elastic modulus of LLAC increased from 0.9GPa to $0.5 \mathrm{GPa}$, the stresses of the LLAC all increased. When the elastic modulus of waterproof increased from $0.15 \mathrm{GPa}$ to $0.45 \mathrm{GPa}$, the shear stress $\sigma_{z \max }$ of the LLAC increased slightly, other stresses of the LLAC all decreased.

When the elastic modulus of the ULAC increased, the part stresses of ULAC increased, the part stresses of ULAC decreased, the stresses of the LLAC all decreased slightly. When the elastic modulus of the LLAC increased, the part stresses of ULAC increased, the part stresses of ULAC decreased, the stresses of the LLAC all increased. When the elastic modulus of the WL increased, the tensile stress $\sigma_{x \max }, \sigma_{y \max }$, shear stress $\tau_{x y \max }$ of the ULAC and LLAC all decreased obviously.

As the vehicle load acted chronically and repeatedly on the asphalt concrete pavement, adjacent layers might have disengaging area. Assumed that the disengaging area existed under A1 in fig 2, and four types of disengaging area were shown in fig 7 . The disengaging area might occur between the ULAC and LLAC, and it also might occur between the LLAC and WL. In consideration of effect of vehicle braking action, the stress value of asphalt concrete pavement were calculated in two cases, one was without vehicle braking action, and the other was under vehicle braking action.

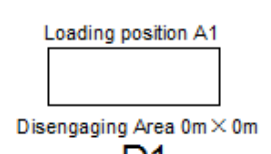

D1

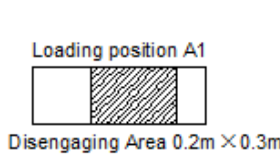

D2

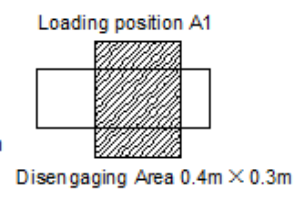

D3

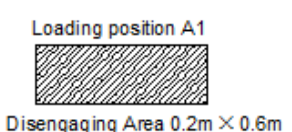

D4

Fig. 7 four types of disengaging area

Stress value of asphalt concrete pavement without vehicle braking action

When vehicle located at the position shown in the fig 2 and without braking action, the stresses of the ULAC under different type of disengaging area were listed in tab 2. The stresses of the ULAC under the type D1 were smaller than the stresses of ULAC under other three types of disengaging area; as the disengaging area under the loading position increased, the stresses of the ULAC also increased; compared with the stresses of the ULAC when the disengaging area occurred between the LLAC and the WL, the stresses of the ULAC when the disengaging area occurred between the ULAC and LLAC were bigger than them. 
Table 2 Stresses of the ULAC under Different Types of Disengaging Area (Unit: MPa)

\begin{tabular}{|c|c|c|c|c|c|c|c|}
\hline & $\begin{array}{c}\text { Disengaging } \\
\text { type }\left[\mathrm{m}^{2}\right]\end{array}$ & $\sigma_{x \max }$ & $\sigma_{y \max }$ & $\sigma_{z \max }$ & $\tau_{x y \max }$ & $\tau_{y z m a x}$ & $\tau_{x z \max }$ \\
\hline \multirow{4}{*}{$\begin{array}{c}\text { Disengaging Area } \\
\text { between the ULAC } \\
\text { and the LLAC }\end{array}$} & $\mathrm{D} 1: 0 \times 0$ & 0.382846 & 0.278042 & 0.110501 & 0.177801 & 0.0940003 & 0.219861 \\
\hline & D2:0.2×0.3 & 0.847505 & 0.930729 & 0.83204 & 0.400471 & 0.286375 & 0.40138 \\
\hline & D3:0.4×0.3 & 1.71697 & 2.73295 & 0.644633 & 0.657533 & 0.547069 & 0.447882 \\
\hline & D4:0.2×0.6 & 1.15193 & 1.16311 & 0.562838 & 0.579082 & 0.356798 & 0.325335 \\
\hline \multirow{4}{*}{$\begin{array}{l}\text { Disengaging Area } \\
\text { between the LLAC } \\
\text { and the WL }\end{array}$} & D1:0 $\times 0$ & 0.382846 & 0.278042 & 0.110501 & 0.177801 & 0.0940003 & 0.219861 \\
\hline & $\mathrm{D} 2: 0.2 \times 0.3$ & 0.503386 & 0.569577 & 0.120668 & 0.346176 & 0.182161 & 0.283791 \\
\hline & D3:0.4×0.3 & 0.789981 & 0.690289 & 0.134943 & 0.496351 & 0.199285 & 0.352494 \\
\hline & D4:0.2×0.6 & 0.704127 & 0.828375 & 0.111312 & 0.459972 & 0.254129 & 0.310126 \\
\hline \multicolumn{8}{|c|}{ Table 3 Stresses of the LLAC under Different Types of Disengaging Area (Unit: MPa) } \\
\hline & $\begin{array}{c}\text { Disengaging } \\
\text { type }\left[\mathrm{m}^{2}\right]\end{array}$ & $\sigma_{x \max }$ & $\sigma_{y \max }$ & $\sigma_{z \max }$ & $\tau_{x y \max }$ & $\tau_{y z \max }$ & $\tau_{x z \max }$ \\
\hline \multirow{4}{*}{$\begin{array}{l}\text { Disengaging Area } \\
\text { between the ULAC } \\
\text { and the LLAC }\end{array}$} & $\mathrm{D} 1: 0 \times 0$ & 0.329191 & 0.584576 & 0.0571854 & 0.203143 & 0.133247 & 0.133619 \\
\hline & D2:0.2×0.3 & 0.617912 & 0.60949 & 0.785231 & 0.238206 & 0.224956 & 0.181073 \\
\hline & D3:0.4×0.3 & 0.74486 & 0.617498 & 0.530226 & 0.323254 & 0.250194 & 0.185011 \\
\hline & D4:0.2×0.6 & 0.402186 & 0.604174 & 0.49014 & 0.203116 & 0.147112 & 0.191644 \\
\hline \multirow{4}{*}{$\begin{array}{l}\text { Disengaging Area } \\
\text { between the LLAC } \\
\text { and the WL }\end{array}$} & D1:0×0 & 0.329191 & 0.584576 & 0.0571854 & 0.203143 & 0.133247 & 0.133619 \\
\hline & D2: $0.2 \times 0.3$ & 1.17202 & 1.56723 & 0.174787 & 0.316017 & 0.285096 & 0.182197 \\
\hline & D3:0.4×0.3 & 1.61783 & 2.02584 & 0.171614 & 0.415267 & 0.293845 & 0.283021 \\
\hline & D4:0.2×0.6 & 1.39087 & 1.93461 & 0.0724281 & 0.457912 & 0.369323 & 0.220842 \\
\hline
\end{tabular}

When vehicle located at the position shown in the fig 2 and without braking action, the stresses of the LLAC under different type of disengaging area were listed in tab 3 . The stresses of the LLAC under the type D1 were smaller than the stresses of LLAC under other three types of disengaging area; as the disengaging area under the loading position increased, the stresses of the LLAC also increased; compared with the stresses of the LLAC when the disengaging area occurred between the ULAC and LLAC, the stresses of the LLAC when the disengaging area occurred between the LLAC and the WL were bigger than them. From tab 2 and tab 3, when the size of the disengaging area increased, the stresses of the ULAC and LLAC all increased; when the size of the disengaging area was the same in D3 and D4, the position of the disengaging area also affected the stresses of the ULAC and the LLAC.

stress value of asphalt concrete pavement under vehicle braking action

When vehicle located at the position shown in the fig 2 and with braking action, the stresses of the ULAC under different type of disengaging area were listed in tab 4. The stresses of the ULAC under the type D1 were smaller than the stresses of ULAC under other three types of disengaging area; as the disengaging area under the loading position increased, the stresses of the ULAC also increased; compared with the stresses of the ULAC when the disengaging area occurred between the LLAC and the WL, the stresses of the ULAC when the disengaging area occurred between the ULAC and LLAC were bigger than them. Compared with tab 2 and tab 4, when the disengaging area occurred between the ULAC and the LLAC, the tensile stresses of the ULAC increased averagely by $6.25 \%$, and the shear stresses of the ULAC increased averagely by $25.48 \%$; when the disengaging area occurred between the LLAC and the WL, the tensile stresses of the ULAC increased averagely by $18.81 \%$, and the shear stresses of the ULAC increased averagely by $41.71 \%$. Compared with the stresses of the ULAC when the disengaging area occurred between the ULAC and the LLAC, the stresses of the ULAC increased more than them when the disengaging area 
occurred between the LLAC and the WL.

Table 4 Stresses of the ULAC under Different Types of Disengaging Area (Unit: MPa)

\begin{tabular}{|c|c|c|c|c|c|c|c|}
\hline & $\begin{array}{c}\text { Disengaging } \\
\text { type }\left[\mathrm{m}^{2}\right]\end{array}$ & $\sigma_{x \max }$ & $\sigma_{y \max }$ & $\sigma_{z \max }$ & $\tau_{x y \max }$ & $\tau_{y z \max }$ & $\tau_{x z \max }$ \\
\hline \multirow{4}{*}{$\begin{array}{l}\text { Disengaging Area } \\
\text { between the ULAC } \\
\text { and the LLAC }\end{array}$} & $\mathrm{D} 1: 0 \times 0$ & 0.433301 & 0.589981 & 0.110954 & 0.317844 & 0.242681 & 0.29957 \\
\hline & $\mathrm{D} 2: 0.2 \times 0.3$ & 0.845104 & 1.14819 & 0.833989 & 0.622343 & 0.428738 & 0.498335 \\
\hline & D3:0.4×0.3 & 1.71593 & 2.71901 & 0.642719 & 0.841229 & 0.635717 & 0.478091 \\
\hline & D4: $0.2 \times 0.6$ & 1.15184 & 1.50169 & 0.589231 & 0.842484 & 0.399093 & 0.332592 \\
\hline \multirow{4}{*}{$\begin{array}{l}\text { Disengaging Area } \\
\text { between the LLAC } \\
\text { and the WL }\end{array}$} & $\mathrm{D} 1: 0 \times 0$ & 0.433301 & 0.589981 & 0.110954 & 0.317844 & 0.242681 & 0.29957 \\
\hline & $\mathrm{D} 2: 0.2 \times 0.3$ & 0.644373 & 0.851329 & 0.121147 & 0.496352 & 0.314126 & 0.36623 \\
\hline & D3:0.4×0.3 & 0.945162 & 0.887494 & 0.135389 & 0.650314 & 0.328066 & 0.432847 \\
\hline & D4:0.2×0.6 & 0.761451 & 1.113 & 0.111743 & 0.614673 & 0.38684 & 0.391531 \\
\hline \multicolumn{8}{|c|}{ Table 5 Stresses of the LLAC under Different Types of Disengaging Area (Unit: MPa) } \\
\hline & $\begin{array}{c}\text { Disengaging } \\
\text { type }\left[\mathrm{m}^{2}\right]\end{array}$ & $\sigma_{x \max }$ & $\sigma_{y \max }$ & $\sigma_{z \max }$ & $\tau_{x y \max }$ & $\tau_{y z \max }$ & $\tau_{x z \max }$ \\
\hline \multirow{4}{*}{$\begin{array}{l}\text { Disengaging Area } \\
\text { between the ULAC } \\
\text { and the LLAC }\end{array}$} & D1:0×0 & 0.345255 & 0.595801 & 0.05733 & 0.150362 & 0.211155 & 0.146043 \\
\hline & $\mathrm{D} 2: 0.2 \times 0.3$ & 0.614065 & 0.602495 & 0.7857 & 0.19316 & 0.325084 & 0.222311 \\
\hline & D3:0.4×0.3 & 0.741358 & 0.595823 & 0.530525 & 0.281249 & 0.37952 & 0.222279 \\
\hline & D4:0.2×0.6 & 0.396539 & 0.595818 & 0.504775 & 0.158798 & 0.226191 & 0.221943 \\
\hline \multirow{4}{*}{$\begin{array}{l}\text { Disengaging Area } \\
\text { between the LLAC } \\
\text { and the WL }\end{array}$} & D1:0×0 & 0.345255 & 0.595801 & 0.05733 & 0.150362 & 0.211155 & 0.146043 \\
\hline & $\mathrm{D} 2: 0.2 \times 0.3$ & 1.16903 & 1.55621 & 0.174232 & 0.286734 & 0.348974 & 0.207533 \\
\hline & D3:0.4×0.3 & 1.61391 & 2.01444 & 0.182715 & 0.360028 & 0.335492 & 0.304953 \\
\hline & D4: $0.2 \times 0.6$ & 1.3882 & 1.92301 & 0.0724849 & 0.398542 & 0.435954 & 0.233746 \\
\hline
\end{tabular}

When vehicle located at the position shown in the fig 2 and with braking action, the stresses of the LLAC under different type of disengaging area were listed in tab 5. The stresses of the LLAC under the type D1 were smaller than the stresses of LLAC under other three types of disengaging area; as the disengaging area under the loading position increased, the stresses of the ULAC also increased; compared with the stresses of the LLAC when the disengaging area occurred between the ULAC and LLAC, the stresses of the LLAC when the disengaging area occurred between the LLAC and the WL were bigger than them. Compared with tab 3 and tab 5, when the disengaging area occurred between the ULAC and the LLAC, the tensile stresses of the LLAC decreased averagely by $0.6 \%$, and the shear stresses of the LLAC increased averagely by $17.22 \%$; when the disengaging area occurred between the LLAC and the WL, the tensile stresses of the LLAC increased averagely by $0.41 \%$, and the shear stresses of the LLAC increased averagely by $5.18 \%$. Compared with the shear stresses of the LLAC when the disengaging area occurred between the ULAC and the LLAC, the shear stresses of the LLAC increased more than them when the disengaging area occurred between the LLAC and the WL.

\section{Conclusions}

(1) Properly increasing the thickness of the ULAC, the most stresses of the ULAC and the LLAC would decreased, the thickness of the ULAC was advised to be $0.04 \mathrm{~m} \sim 0.06 \mathrm{~m}$, and the thickness of the LLAC was advised to be $0.05 \mathrm{~m} \sim 0.07 \mathrm{~m}$. (2) Increasing the elastic modulus of the ULAC and the LLAC, the stresses of themselves increased, but increasing the elastic modulus of the WL, the tensile stress $\sigma_{x \max }, \sigma_{y \max }$, shear stress $\tau_{x y \max }$ of the ULAC and LLAC all decreased obviously. (3)

When the disengaging area occurred between two adjacent layers, the total stresses of the ULAC 
and the LLAC would all increase. The vehicle braking action had a great effect to the stresses of the ULAC when the disengaging area occurred between two adjacent layers. (4) When the disengaging area occurred between the ULAC and LLAC, the stresses of the ULAC changed obviously. When the disengaging area occurred between the LLAC and the WL, the stresses of the LLAC changed obviously. (5) The stresses of the ULAC and the stresses of the LLAC would change with the size of the disengaging area, and would also change with the position of the disengaging area.

\section{Acknowledgements}

This research is an outgrowth of a study sponsored by Natural Science Basic Research Plan in Shaanxi Province of China (Program No. 2013JM7002).

\section{References}

[1] Wu Bo: Southwest Jiaotong University (2015)

[2] Xiao Xingqiang: submitted to Journal of Chongqing Jiaotong University(Natural Science) (2008)

[3] HU Chunhua: submitted to American Society of Civil Engineers (2012)

[4] QI Wenyang: submitted to Journal of East China Jiaotong University (2014)

[5] Kruntcheva M R, Collop A C: submitted to American Society of Civil Engineers (2006)

[6] XU Qinwu, HU Changshun and WANG Hu: submitted to Journal of Chang'an University (Natural Science Edition) (2007)

[7] HU Xiao: submitted to Highway Engineering ( 2007)

[8] Zhou Yumin, Tan Zhiming: GeoShanghai International Conference 2010 (2010)

[9] LI Na, HAN Sen, YU Jingtao: Proceedings of the 10th International Conference of Chinese Transportation Professionals (2010)

[10] AI Changfa, QIU Yanjun, MAO Cheng: International Conference on Transportation Engineering (2007)

[11]Wang Xinmin: ANSYS Structural Analysis Unit and Application (China Communications Press, Beijing 2009)

[12] CCCC Highway Planning and Design Institute: Ministry of Communications of the People's Republic of China (2004) 